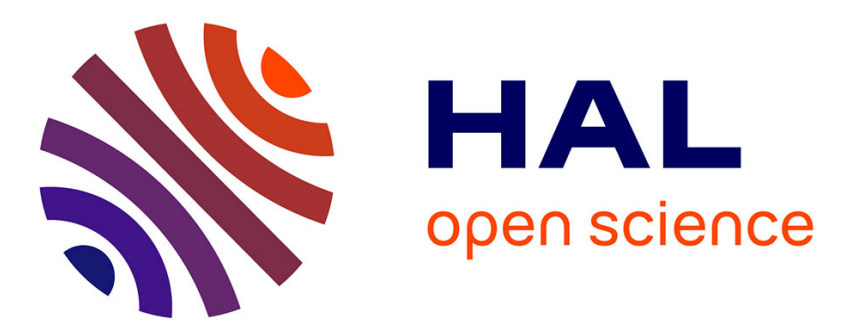

\title{
Experiments of draining and filling processes in a collapsible tube at high external pressure
}

\author{
P. Flaud, P. Guesdon, J.-M. Fullana
}

\section{To cite this version:}

P. Flaud, P. Guesdon, J.-M. Fullana. Experiments of draining and filling processes in a collapsible tube at high external pressure. European Physical Journal: Applied Physics, 2012, 57 (3), 10.1051/epjap/2012110234 . hal-00793783

\section{HAL Id: hal-00793783 \\ https://hal.science/hal-00793783}

Submitted on 23 Feb 2013

HAL is a multi-disciplinary open access archive for the deposit and dissemination of scientific research documents, whether they are published or not. The documents may come from teaching and research institutions in France or abroad, or from public or private research centers.
L'archive ouverte pluridisciplinaire HAL, est destinée au dépôt et à la diffusion de documents scientifiques de niveau recherche, publiés ou non, émanant des établissements d'enseignement et de recherche français ou étrangers, des laboratoires publics ou privés. 


\title{
Experiments of draining and filling processes in a collapsible tube
}

\section{at high external pressure}

\author{
P. Flaud ${ }^{1}$, P. Guesdon ${ }^{2}$, and J.-M. Fullana ${ }^{3}$ \\ 1 - Matière et Systèmes Complexes \\ Université Paris 7 \\ 2 - Boston Scientific \\ 3 - Institut Jean Le Rond d'Alembert \\ UPMC
}

January 24, 2012

\begin{abstract}
The venous circulation in the lower limb is mainly controlled by the muscular action of the calf. To study the mechanisms governing the venous draining and filling process in such situation, an experimental setup, composed by a collapsible tube under external pressure has been built. A valve preventing back flows is inserted at the bottom of the tube, and allows to model two different configurations : physiologic when the fluid flow is uni-directional and pathological when the fluid flows in both directions. Pressure and flow rate measurements are done at the inlet and outlet of the tube and an original optical device with three cameras is proposed to measure the instantaneous cross-sectional area. The experimental results (draining and filling with physiologic or pathological valves) are confronted to a simple one dimensional numerical model which completes the physical interpretation. One major observation is that the muscular contraction induces a fast emptying phase followed by a slow one controlled by viscous effects, and that a defect of the valve decreases, as expected, the ejected volume.
\end{abstract}

\section{Introduction}

Veins have flexible walls that can support, in normal situations like walking or breathing, high external pressures. Veins are deformable, which means they may collapse when the internal pressure falls below the external pressure. Venous collapse is an important physiological mechanism of the low leg blood circulation. Indeed, (i) it helps the blood getting back to the heart and (ii) it is one of the targets in the therapies for the prevention and treatment of chronic 
venous insufficiency (CVI) and deep venous thrombosis (DVT). Venous collapse results from a complex nonlinear interaction between venous pressure, blood flow rate and the mechanics of the vessel walls, which can lead to a variety of phenomena, e.g. flow limitation, self oscillations, or wave propagation.

Studies on collapsible tubes are an important element of the venous vessels fluid dynamics and of the fluid-structure interaction between blood flow and venous walls. Experimental setups consisting in a collapsible tube under external pressure provide a simple system allowing to model and study blood flow. These experimental flows are complex enough to describe properly the fluid-structure interaction and nevertheless allow measuring the dynamic variables (i.e. flow rate, tube area, internal and external pressures). In the area of theoretical developments, simple onedimensional mathematical models were shown consistent with the experimental observations; Shapiro [1] presented an analysis the one-dimensional steady flow through a collapsible tube (including friction, gravity and variations of the external pressure), and many other works proposed unsteady and steady numerical solutions of one-dimensional flows [2-4].

The experimental flow analysis using collapsible tubes has been developed simultaneously with the investigation of biological flows, such as the venous blood flow, the pulmonary air flow and the periodic external compression (foot boots, breathing help). Numerous studies handle with flow through elastic and collapsible tubes, applied, for instance, to arteries, veins, bronchi or urethrae [5], [6],
[7], [3], [4], [8]. Phenomena like flow limitation in Starling resistor $([9],[10])$ with a transition between super-critical and sub-critical flow was observed. Indeed, almost all experimental setups rely on the Starling classical setup consisting of a horizontal tube with given external pressure since it allows the emergence of super-critical phenomena. The influence of time-dependent conditions is dealt with in [11] [2] [12]. Flow separation near a constriction was studied numerically by [13] [14] [15]. Recent research provides numerous examples of experimental studies about tube oscillations [16] [17] [18] [19].

In medical applications, researchers did not pay much attention to venous blood circulation partially because the physiologists have long considered the venous network just as a blood reservoir. Nevertheless, there is physiological evidence that the mechanics of the solid-fluid coupling, such as the muscular activity and its interaction with the blood flow, or valve failure, have an important effect on the blood circulation.

The muscular pump is the main mechanism explaining the decrease of the Ambulatory Venous Pressure (AVP). The AVP is the principal functional index used to assess the degree of malfunctioning of the lower leg venous system: the pressure variations are recorded at the distal Marginal Vein (superficial veins located at the upper surface of the foot) under muscular activity (i.e. walking, rising up tiptoe) and, in a healthy leg, the pressure decreases with each muscular pumping cycle. When there is a deficiency in the muscular pump or a valvular incontinence, the AVP rises and produces an ambulatory hyper-pressure 
and a short refilling time. The refilling time, defined as the characteristic time required to reach the AVP baseline after muscular activity, is another important marker of illness. In summary, in this context severe illness is correlated with high AVP and with short refilling time.

Muscular veins of the lower leg (which are inside muscular compartments) support high external pressure when the muscular pump is activated and they provide a good framework to study, experimentally and numerically, the non-linear interactions between high external pressure and venous flow. The diameters of the muscular veins are relatively large compared to the typical vein diameters of the lower leg, thus simplifying the dynamics similarity for the experimental setup (because a large diameter allows to adjust viscosity conveniently).

In this paper, we present an experimental setup to model the draining and filling processes of the muscular veins of the lower leg: a collapsible tube was placed vertically inside a hermetic chamber connected to a tank with controlled air pressure. Inside the chamber, the tube was surrounded by air. The controlled experimental setup allows a very quick switch from high positive to negative transmural pressure ${ }^{1}$, as observed during muscular activity.

The use of an electrovalve allows studying two modes of draining and filling: the normal intramuscular venous system function where the fluid flows only in the upper direction, and a crude picture of the calf pump failure that

\footnotetext{
1 The "transmural pressure" is the difference between the internal and the external pressures.
}

happens when the fluid flows in both directions. This experimental setup provides a variety of interesting phenomena observed in very viscous systems, such as propagative waves or a quasi steady draining. The experimental data were compared to the results of an one-dimensional numerical model.

The paper is organised in three sections. Section 2 presents the experimental setup as well as the material and the protocols used for pressure, flow rate and crosssectional area measurements. Section 3 presents the experimental results of the draining and the filling processes.

We use the numerical simulations in Section 4 to improve the physical interpretations.

\section{Experimentation}

\subsection{Experimental setup}

Figure 1 shows the experimental setup, which consists in three chambers, upper, central and bottom, with a collapsible tube inside the central chamber that connects the upper and the bottom chamber. The fluid is located in the hatched region. A secondary circuit (on the left of the Figure 1) directly relies the upper and the bottom chambers. The connection between both flow circuits is managed by an electrovalve (EV) that controls the back-flows.

The central chamber is hermetic and tight to air and water; it is made in dural (an equilateral triangle of 100 $\mathrm{mm}$ of side length and of $2.5 \mathrm{~mm}$ of thickness) with longitudinal windows of about $500 \mathrm{~mm}$ that allow measuring the tube deformation. The area variations $A(t)$ result 


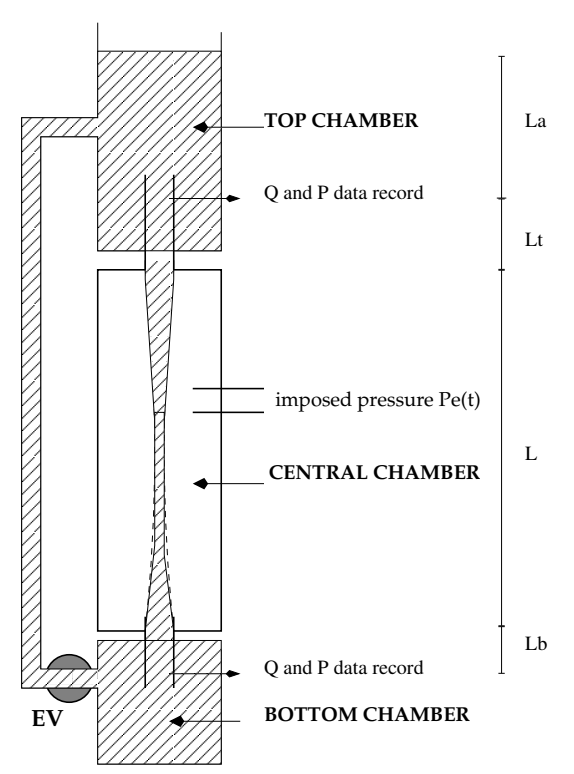

Fig. 1. Scheme of the experimental setup composed of three chambers: upper, central and bottom. The central chamber contains a vertical collapsible tube that connects the upper and the bottom chamber. The fluid is located in the hatched region. The pressure $P_{e}(t)$ acts like the calf muscular pump. The electrovalve (EV) controls the back-flows into the secondary circuit.

from the computation of the cross-sectional area of the tube by image processing. The windows are chemically treated with black anodic oxidation to minimize the laser reflections used in the area reconstruction protocol. The central chamber is also connected to an external tank filled with compressed air $\left(P_{e}(t)\right.$ in the Figure 1$)$. This device controls the pressure inside the chamber and thus outside the collapsible tube. The external pressure $P_{e}(t)$ was considered a model of the intramuscular pressure during muscular activity.

Inside the central chamber, a collapsible tube is attached to rigid tubes of similar internal diameter. The rigid tubes connect the collapsible tube to the top and the bottom chambers respectively. In these two rigid tubes, the pressures $\left(P_{t o p}\right.$ and $\left.P_{b o t}\right)$ and the flow rate $\left(Q_{t o p}\right.$ and $\left.Q_{b o t}\right)$ were measured by pressure sensors and Ultra-Sound (US) Doppler respectively.

The bottom chamber also includes an on-off electrovalve EV to model the valvular system of the venous network. The electrovalve's response time was measured to be less than $10 \mathrm{~ms}$. According to the healthy function of the intramuscular venous system, the "closed" mode allows the fluid to flow only in the upper direction, the bottom chamber being disconnected from the external circuit. In the "open" mode, the experimental configuration corresponds to the calf pump failure: the fluid can flow in both directions.

The collapsible tube is placed vertically into the central chamber and is $L=39 \mathrm{~cm}$ long. The relative position of the two rigid tubes is adaptable; this mechanism allows changing the longitudinal tube tension by its length variation. The collapsible tube is made in natural rubber obtained by molding, a technical procedure which ensures a homogeneous thickness. We used several tubes of 8, 10 and $12 \mathrm{~mm}$ of diameter with variable thickness $(2 / 10,3 / 10$ and 4/10 $\mathrm{mm}$ ). These diameters and thickness were chosen according to the dynamical similitude, as stated above. The experimental results presented in this paper correspond to a tube with diameter $12 \mathrm{~mm}$ and thickness $2 / 10 \mathrm{~mm}$.

Table 1 presents the main characteristics of the experimental configuration: $L$ is the length of the collapsible tube, $L_{t}$ and $L_{b}$ are the relative distances between the measuring points in the rigid tube and upper and lower 


\begin{tabular}{c|c|c|c|c} 
Lt & L & Lb & A0 & Rigid diameter \\
\hline $37.5 \mathrm{~cm}$ & $39 \mathrm{~cm}$ & $39.5 \mathrm{~cm}$ & $1.12 \mathrm{~cm}^{2}$ & $1.2 \mathrm{~cm}$
\end{tabular}

Table 1. Experimental data. Lt, L and Lb are the lengths in $\mathrm{cm}$.

edges of the collapsible tube, $A 0$ is the neutral tube area (the cross-sectional area for zero transmural pressure) and the last column gives the diameter of the rigid tubes. The Reynolds number is in the range 1500-2000.

\subsection{Measurement of the pressure}

To measure the pressure under flow conditions we used a strain-gauge type of transducer (OMICRON, type OMP02). The pressure applied on the membrane generates micro deformations inducing a resistance variation on the straingauge and a signal that is proportional to the pressure. The range of pressure measurements was from 0 to 100 $\mathrm{kPa}$ with a precision of $0.1 \%$.

\subsection{Measurement of the flow rate}

Two US Doppler probes were placed in the top and bottom chambers and connected to the two rigid tubes. They were calibrated at constant flow rate using a DOP 1000 velocimeter and $5 \mathrm{MHz}$ and $10 \mathrm{MHz}$ probes. The US Doppler measured the velocity at different radial positions, and gave directly the velocity profile. Assuming an axi-symmetric the tube axis, a pre-calibrated grid with equi-spaced black flow, we calculated the flow rate by a numerical integration of the velocities measured in the proximal half tube diameter, where the signal-to-noise ratio level is satisfactory.

\subsection{Fluid}

The fluid used in the experiment is an aqueous solution composed of glycerol at $60 \%$ and echogenic Rilsan particles of average diameter 20 to $30 \mu \mathrm{m}$ in suspension. In preliminary experiments of flow rate calibration using the US Doppler (DOP 1000), we have found that for a concentration of $140 \mathrm{mg} / \mathrm{L}$ of Rilsan particles we got a precise evaluation of the velocity profile. The physical properties of the fluid at ambient temperature were : a viscosity of $1310^{-3} \mathrm{~Pa} s$ and a density of $1100 \mathrm{Kg} \mathrm{m}^{-3}$. Recall that blood viscosity range belongs to $[4: 100] 10^{-3} \mathrm{~Pa}$ s. The fluid fulfills the similarity requirements.

\subsection{Optical device for area measurement}

External to the central chamber an optical device includes a thin laser sheet and three high-definition cameras (CCD). The laser sheet intercepts the collapsible tube generating the measured images. The cameras were put all in the same horizontal plane and separated by 120 degrees. Each camera was coupled to a polarimeter to adjust the contrast. For a good reconstruction it was necessary, for each image, to be placed in a plane that is perpendicular to the tube axis. To take into account the image distortion induced by the vertical angle between the camera and points disposed in two dimensions was put in that plane before every experiment. In a post-treatment procedure the geometrical relationships between the grid points gave the angular corrections for each camera. The acquisition 


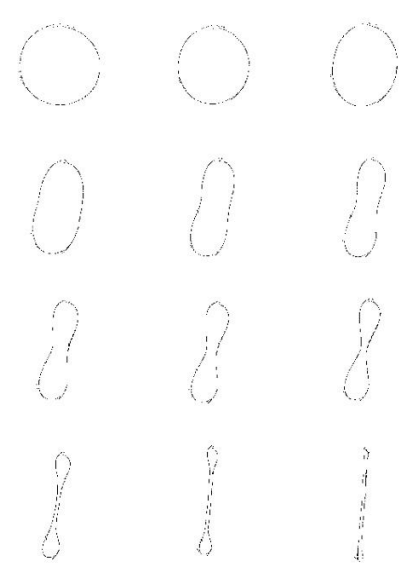

Fig. 2. Example of reconstructed images from the three CCD cameras during a collapse process with increasing time from top-left to bottom-right

frequency was chosen between 60 and 120 images/second depending on the nature of the experiment. Data analysis of the three images including a fitting procedure allowed to compute the tube area with an error of only a few square pixels. The experimental measurements of cross-sectional area were done in a static configuration (a filled elastic tube with no flow). This implies that the visco-elastic and the inertial effects of the wall were neglected when we have built the experimental tube law.

The Figure 2 shows an example of the evolution in time of the reconstructed cross-sectional area of the elastic tube during a collapse process, with increasing time from topleft to bottom-right. The cross-sectional area of the tube depends on the transmural pressure $\left(p_{i}-p_{e}\right)$. The tube collapse follows a well known dynamics from a slightly oval cross-sectional area to a line contact.
We have compared these results with those obtained by the ombroscopy technique. In this later technique, an emitter sends a laser sheet in the tube direction. The receptor has a photosensitive cell that evaluates the shadow zone delimited by the tube shape corresponding to the tube apparent diameter. The area evaluation is obtained by two orthogonal measurements of the minor $D_{s}$ and the major $D_{g}$ tube apparent diameter. The value of the tube area is then computed using the ellipticity hypothesis by $A=\pi D_{s} D_{g} / 4$

At positive transmural pressure the tube is inflated and the section is circular. In this case the two methods give the same results. When the transmural pressure becomes negative the tube is partially collapsed. The area values computed by the two methods present remarkable differences mainly beyond the contact point. The ellipticity hypothesis breaks down when the lobes appear [20], and the ombroscopy technique then overestimates the tube area. This detail is critical for the numerical comparisons of the experiment since it underestimates the viscous term of the numerical model of Section 4, which is based on an evaluation of the cross-sectional area.

This appears clearly when we consider the tube law $F_{\text {exp }}$, which relies the non dimensional area $A / A_{0}$ with the transmural pressure $p_{t}=p_{i}-p_{e}=F_{\text {exp }}\left(A / A_{0}\right)$, where $A_{0}$ is the cross-sectional area at zero transmural pressure and $p_{i}$ is the internal pressure. In both cases, the determination of $F_{\text {exp }}$ was done at the middle height of the tube, far enough of the fixed ends to eliminate potential errors introduced by these fixations. A detail of the tube 


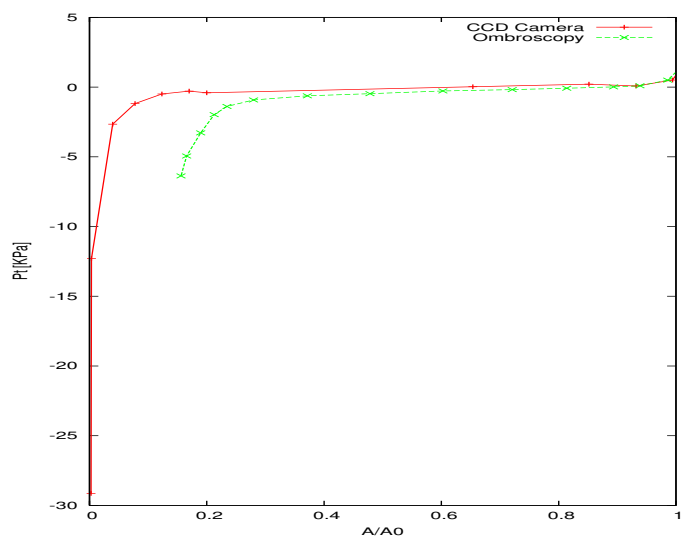

Fig. 3. Experimental tube law, $p_{i}-p_{e}=F_{\text {exp }}\left(A / A_{0}\right)$ at negative transmural pressure. CCD Camera (red line) and ombroscopy (green line)

law at negative transmural pressure obtained with a CCD camera (red line) or ombroscopy (green line) is shown in Figure 3 ; the difference between the vertical axis and the asymptote of the area-pressure relationship directly gives the minimum value of the cross-sectional area when the tube is completely collapsed.

The complete protocol of the procedure is described in reference [21].

\section{Experimental results and discussion}

This section presents the experimental results of the model (flexible tube subjected to high external pressure) of draining and filling process in a muscular pump of the lower leg.

We recall that in a normal configuration the venous valves are continent and the blood flows a single direction, carrying the blood towards the heart. On the other hand, in a pathological process, the venous valves are partially open and the blood can flow in both directions. For each situation, we present the experimental results of two modes : (i) open (electrovalves open, a model of pathological process) and (ii) closed (electrovalves closed, a model of normal or healthy process).

\subsection{Draining a tube}

At the initial condition, the elastic tube is completely filled and the system is in a static configuration, i.e., zero flow rate and constant pressure. To start the process we set the external pressure $P_{e}(t)$ and we compute the velocities and the pressures.

\subsubsection{Draining open mode - (pathological model)}

Figure 4 presents the experimental results of the draining process for the open mode: the fluid can flow towards the top and bottom chambers from both sides of the collapsible tube. The results show

- (a) the pressures [kPa]: red - external (ext), green - top tube (top) and blue - bottom tube (bot),

- (b) the flow rate $\left[\mathrm{cm}^{3} / \mathrm{s}\right]$ : top rigid tube (top) in green and bottom rigid tube (bot) in blue,

- (c) the normalized tube volume $\frac{V(t)}{V(0)}$ giving the ejected fluid volume: top exit (top) in green, bottom exit (bot) in blue, and total ejected volume (total) in red. $V(0)$ is the initial tube volume and $V(t)=V_{t}(t)+V_{b}(t)$ where $V_{t}(t)$ is defined by $V_{t}(t)=\int_{0}^{t} Q_{t}(t) d t$ (similarly for $\left.V_{b}(t)\right)$.

As stated above, before the activation of the external pressure, between 0 and $0.5 \mathrm{~s}$. the experimental system is 

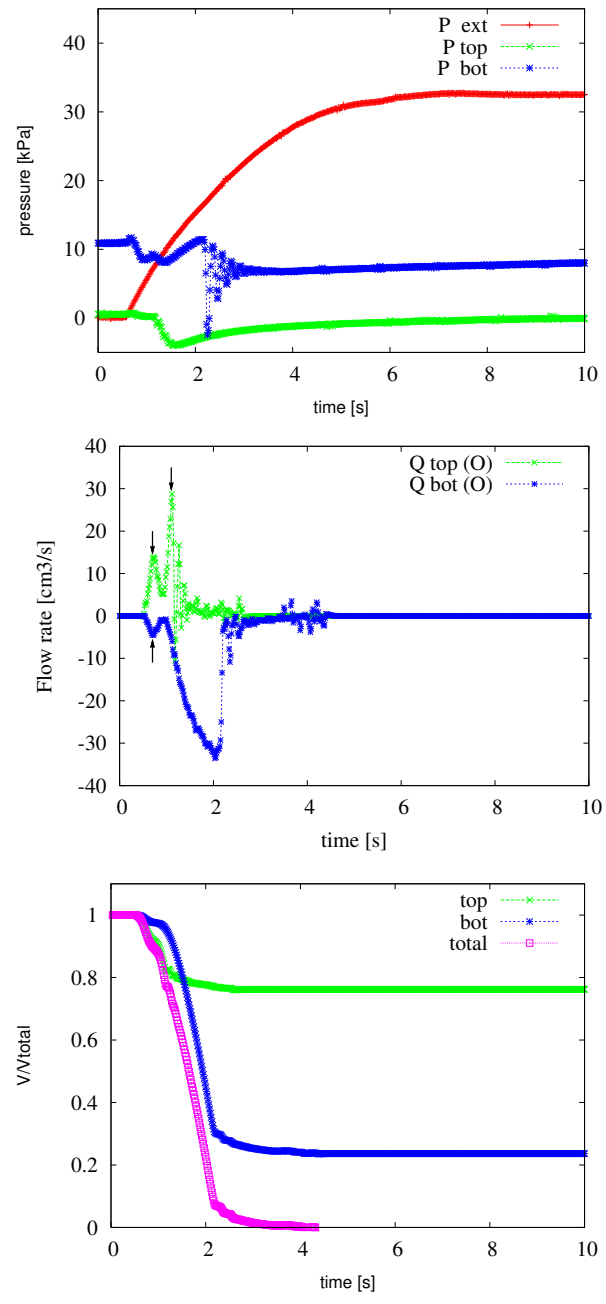

Fig. 4. Experimental results of the draining process, open mode (pathological model) : (a) pressure (external, top tube, and bottom tube), (b) flow rate at top and bottom rigid tube (c) the normalized tube volume.

in a static configuration. Consequently, the pressure sensors measure the hydrostatic pressure and the flow rate is zero. We observe in Figure 4 (a) that the difference of pressures between the pressure sensors at the top and the bottom rigid tubes is the hydrostatic pressure $\rho g(L t+L+L b)$. At the initial time $t_{i}=0.5 \mathrm{~s}$, the external pressure is set up and it reaches its maximum value of around $32 k P a$ at $t \sim 6 s$ (in red in Figure $4(\mathrm{a}))$.
At $t_{i}$, i.e. at the time when the external pressure is applied, the fluid pressure inside the collapsible tube (internal pressure $p_{i}$ ), which cannot be measured, increases quasi instantaneously by the same amount. First, the fluid is accelerated by the pressure gradient which is nonzero mainly at both ends of the elastic tube. Thus the flow rate gradient is nonzero at the ends of the elastic tube. The flow rate $Q$ is related to the time derivative of the crosssectional area $A$ by the continuity relation $\partial_{x} Q+\partial_{t} A$. This explains why we observed, in the early stage of the dynamics, the collapse at both ends of the elastic tube in the zone of fluid acceleration.

The flow rate starts simultaneously with the tube's cross-sectional area decreasing near the ends of the tube, as shown in Figure $4(\mathrm{~b})$ for $t=t_{i}$. The flow rates $Q_{t o p}$ and $Q_{b o t}$ in Figure 4 (b) have an abrupt peak at $t=$ $0.7 s$ (i. e. $0.2 s$ after the beginning of the process) and decrease even though the external pressure is still rising. A second peak appears at $t=1 \mathrm{~s}$ and at $t=2.1 \mathrm{~s}$ for the top and the bottom flow rate respectively. The top flow rate behaviour can be explained by the quickly decrease of the cross-sectional area in the upper part of the tube.

This collapsed zone then extends increasingly towards the bottom, inducing more and more important viscous losses.

During the draining process, we computed, from experimental data, the volume $V(t)$ of the elastic tube as a function of the time. The Figure 4 (c) presents the time evolution of the normalized tube's volume $V(t) / V(0)$ for the top side (green line), the bottom side (blue line) and the total (red line). We observe that the second peak of 
each side arises when the corresponding ejected volume reaches its limit, and that after $t=2.1 \mathrm{~s}$ the elastic tube is quasi-empty.

\subsubsection{Draining close mode (healthy model)}

In the closed mode, the dynamics of the system behaves in two particular ways: (i) when the external pressure ramp is slow enough the system runs into a continuous evolution, the cross-sectional area becomes practically zero proportionally to the pressure signal, (ii) when the pressure ramp is more abrupt, a constriction zone appears near the top end of the elastic tube and the dynamics is in essence different.

Figure 5 presents the experimental results of the draining mode in a closed mode with an abrupt pressure ramp and where the electrovalve at the bottom chamber has been activated thus preventing the fluid to flow to the secondary circuit (circuit parallel to the central chamber in Figure 1).

Experimental results show a very different behavior of the pressure-time variation compared to the open mode situation (Figures 4 (a) and $5(\mathrm{a})$ ). The flow rate $Q_{\text {top }}$ follows a similar time evolution as in the open mode. $\mathrm{Nu}-$ merical simulations in the same configuration presented in Section 4 allow to compute the time evolution of both, the flow rate and the cross-sectional area, and explain the dynamic behavior.
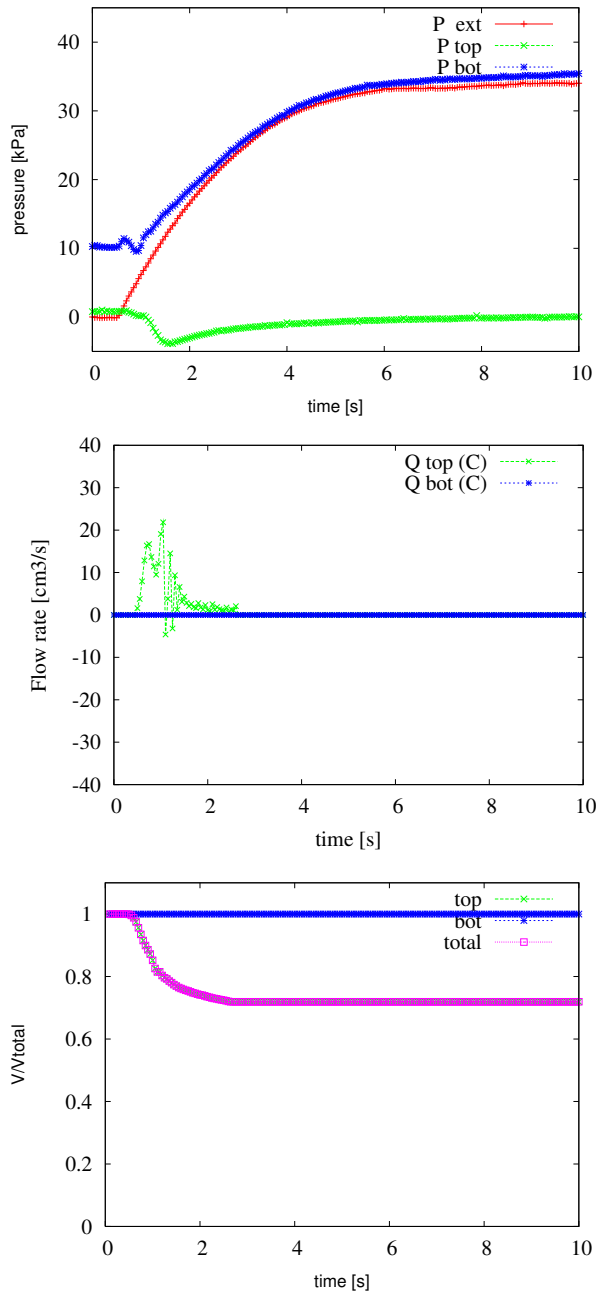

Fig. 5. Experimental results of the draining process, closed mode (healthy model): (a) pressures (external, top tube, and bottom tube), (b) the flow rate at the top and the bottom of the rigid tube, and (c) the normalized tube volume.

\subsection{Filling a tube}

We present the experimental results of the filling process of an elastic tube which is initially completely collapsed. This condition is achieved by setting a high external pressure $P_{e}$, representing the final state of the draining process.

The elastic tube can be filled in by both ends (open mode - pathological model) or by the top end (closed mode - healthy model). 

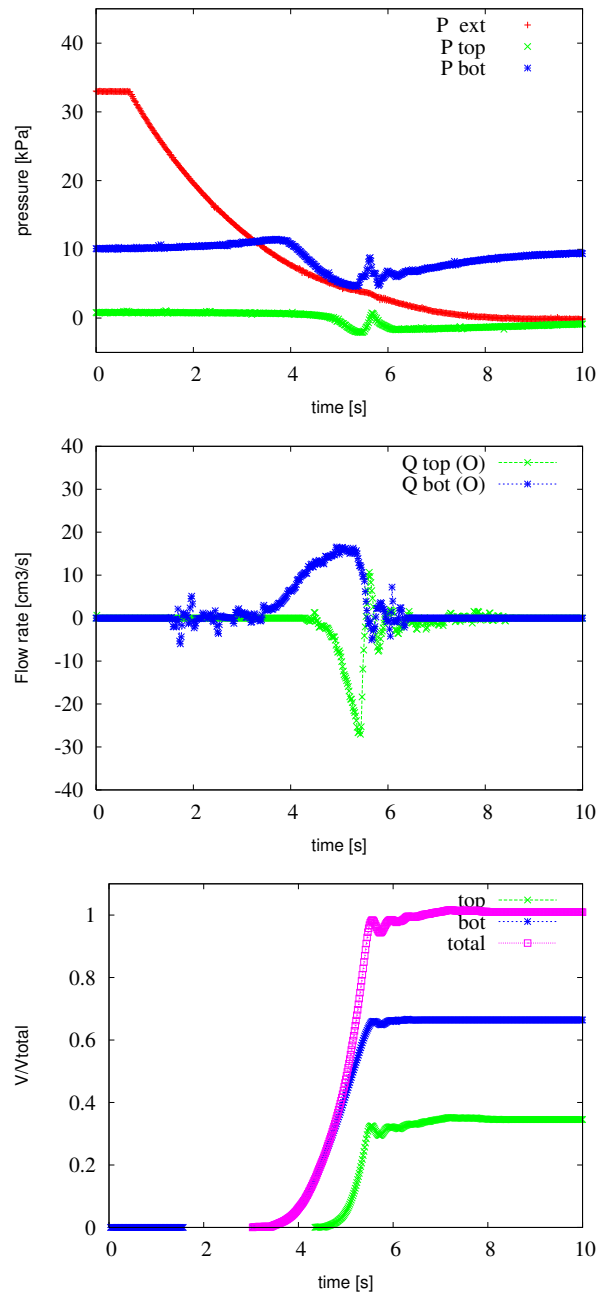

Fig. 6. Experimental results of the filling process, open mode (pathological model) (a) pressure (external, top tube, and bottom tube), (b) the flow rate at the top and the bottom of the rigid tube, and (c) the normalized tube volume.

\subsubsection{Filling open mode (pathological model)}

The time evolution of the measured pressures is shown in Figure 6 (a). We observe that the fluid does not flow while the internal pressure at the top and the bottom ends of the elastic tube does not exceed the reference pressure given by the static configuration. Once the transmural pressure becomes positive, the tube opens and the fluid begins to flow, first at the bottom then at the top of the tube.
The experimental data of the flow rate confirms that the fluid starts to flow at the bottom of the elastic tube (Figure $6(\mathrm{~b})$ ). This is due to the positive transmural pressure at the bottom end which is reached first due to the gravity law. In the top chamber, we have $L a=15 \mathrm{~cm}$ between the free surface and the pressure sensor, hence the pressure at the bottom of the upper rigid tube, $\rho g(L a+L t)$ is around $5.14 k P a$. For the bottom side the pressure is found adding the tube length, which gives about $9 k P a$. The time required for the external pressure to decrease from the pressure at the bottom $\sim 9 \mathrm{kPa}$ to the pressure at the top $(5.15 \mathrm{kPa})$ was about $1.2 \mathrm{~s}$. This time variation corresponds to the difference between the two starting times of the bottom and the top flow rates.

Unlike the draining process, there is only one peak for each flow rate and they appear simultaneously.

\subsubsection{Filling closed mode - (healthy model)}

Similarly to the open mode, the initial condition is an empty elastic tube. Figure 7 (a) presents the time evolution of the three pressures: external tank, rigid top tube and rigid bottom tube. Unlike the open mode, we can observe that at $t=0$ the signal of the bottom sensor does not evaluate the static pressure. The empty tube acts like a membrane and separates the bottom chamber from the upper one. The upper chamber becomes isolated from the external driving pressure and the inflated side of the elastic tube behaves as semi-infinite dynamical system. 

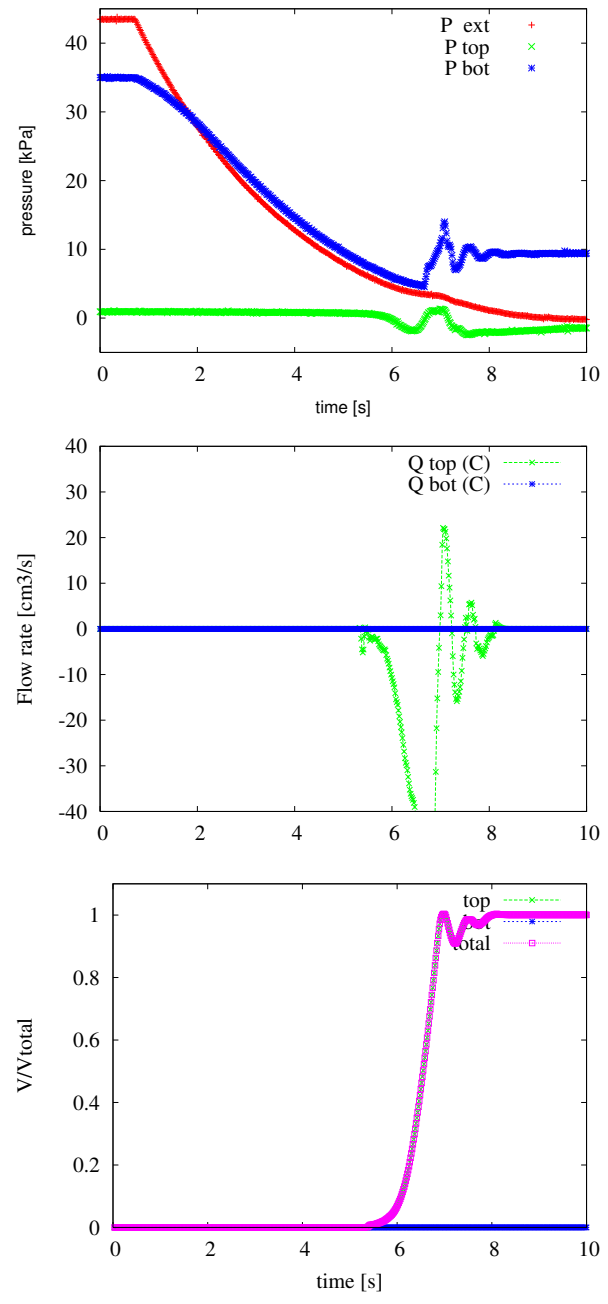

Fig. 7. Experimental results of the filling process, closed mode (healthy model) (a) pressure (external, top tube, and bottom tube), (b) the flow rate at the top and the bottom rigid tube, and (c) the normalized tube volume.

\section{Numerical simulations}

In this section we present the numerical model of a collapsible tube used to add information to the physical understanding of the problem. We assume an unsteady, incompressible one-dimensional flow through a collapsible tube; the variables are then the cross-sectional area $A$, the average velocity $U$ and the internal pressure $p_{i}$. The governing equations for the fluid flow are the conservation of mass [1]

$$
\frac{\partial A}{\partial t}+\frac{\partial}{\partial x}(A U)=0
$$

and the momentum conservation

$$
\rho \frac{\partial U}{\partial t}+\rho U \frac{\partial U}{\partial x}=-\frac{\partial p_{i}}{\partial x}-\rho g-f_{v}
$$

The viscous loss effects are included in the last term $f_{v}$. In our computations we use the semi-analytical expressions based on the thin-shell theory, given in [22]. The mechanical characteristics of the tube are described by the experimental tube law (see Section 2) that relates the transmural pressure $p_{t}=p_{i}-p_{e}$ to the reduced crosssectional area $A / A_{0}$. The experimental tube law is

$$
p_{i}-p_{e}=F_{\text {exp }}\left(A / A_{0}\right)-T / R_{l}
$$

where $T$ is the longitudinal tension and $R_{l}$ is the local longitudinal radius of curvature (positive when the tube cross-section is concave outward). For a thin tube if the fluid density is comparable with the tube density, the inertia of the tube can be neglected [23]. The longitudinal tension $T$ is evaluated directly on the flexible tube by measuring its longitudinal strain.

To close the system we define the boundary conditions at the top $(x=L)$ and at the bottom $(x=0)$ of the tube. The velocities $U(0)$ and $U(L)$ are coupled to a model of the hydraulic circuit. We introduce the mechanical properties of the rigid part of the system: the pressure drop across the rigid tube is modeled by a resistive term and an inertial term. The main contribution to the resistive 
term is classically taken proportional to the square flow rate, and the inertial one is the product of the effective length and the fluid acceleration, thus for $x=0$

$$
p(0, t)=p_{R}-\rho L_{\text {circuit }} \frac{d U}{d t}(0, t)-k L_{\text {circuit }} \rho U^{2}(0, t)
$$

where $p_{R}=\rho g L_{a}$ is the reference pressure or the hydrostatic pressure difference between the free surface of the reservoir at height $L_{a}$ and the origin of the flexible tube, the term $L_{\text {circuit }}$ is the equivalent length, and $k$ is a friction coefficient. The same equation holds for the bottom side at $x=L$ where $p_{R}=\rho g\left(L_{a}+L_{t o p}+L+L_{b o t}\right)$ and $L_{\text {circuit }}$ includes the secondary circuit. The friction coefficient $k$ depends on the shape of the hydraulic circuit. The numerical solutions are computed using a classical integration scheme well-adapted to hyperbolic problems. The MacCormack scheme is a classical two-step predictorcorrector technique, with the following characteristics: it has explicitly conservative form, it is three-points in space, two-levels in time and it is second order accurate in time and space. An approximate solution is obtained in the first step and then corrected in the second. A reference on an evaluation/performance of the MacCormack scheme on collapsible tubes is [24], we already used this scheme on a similar problem [25].

In the following numerical simulations we use the experimental data of pressure $p_{e}(t)$ as an input to our model (3) To compare the numerical and experimental data, we select the numerical results at the corresponding experimental measurement points, i.e., top and bottom of the rigid tubes.
Moreover the numerical data allow us to follow the spatio-temporal dynamics of the cross-sectional area $A$ and the velocity $U$ everywhere and compare them to the specific points of the experimental data.

\subsubsection{Draining open mode (pathological model)}

Figure 8 (top) shows the corresponding numerical solutions of the time evolution of the tube shape in a draining mode. We represent the tube shape for constant time intervals of $0.1 s$ starting from the initial condition $t=t_{i}$ to the second peak observed in the measured flow rate at the bottom of the tube for $t \sim 2.1 s$ (black dotted lines). The red lines represent particular shapes for $t=0.5,0.7,1 ., 2$ which are related to the experimental flow rate peaks of Figure 4 (b). The elastic tube begins to collapse quickly between $t=0.5 \mathrm{~s}$ and $t=0.7 \mathrm{~s}$, quasi uniformly in terms of pressure but not in terms of area because of the non-linear tube law.

At time $t=1 . s$ a second peak appears in the top flow rate $Q_{\text {top }}$. Figure 8 (bottom) shows the comparison between experimental data (green and blue dotted lines as in Figure 4(b)) and numerical simulations (red point-line and line), the flow rate peak at $t=1 . s$ can be regarded as a consequence of the quick area decrease. As above, this time can also be considered as the starting point of (3).the viscous dynamics due to the collapsed region of the tube. At that moment, when the top flow rate stops, the small area of the elastic tube at the upper side acts like a resistive zone and the remaining fluid is forced to flow to the bottom of the elastic tube. At time $t=2.1 \mathrm{~s}$, a second 

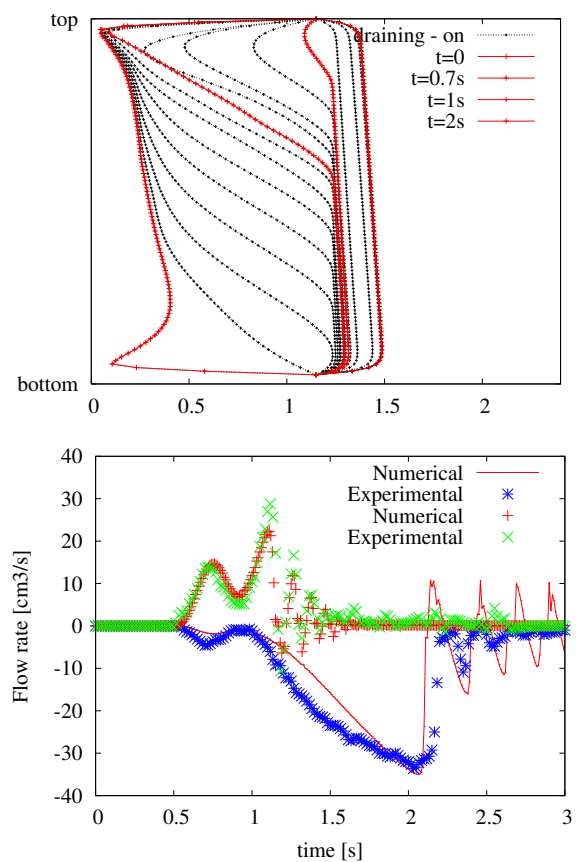

Fig. 8. (top) Numerical results of the draining process. Tube shape (cross-sectional area) at different times. (bottom) Comparison between experimental data and numerical simulations: flow rate as a function of time. (See Fig. 4 (b) for details.)

peak appears in the flow rate at bottom $Q_{b o t}$; Figure 8 (top and bottom) shows that a second zone of viscous dynamics is set up by the narrowing of the elastic tube near the bottom. After $t=2.1 s$, when oscillations appear in experimental results, the dynamics follows a viscous draining. Numerical oscillations in the viscous draining are larger than those observed in experimental data; we recall that the numerical model is parameter free, and we use the numerical solution as black box to understanding the dynamics.

\subsubsection{Draining close mode (healthy model)}

Figure 9 (top) presents the numerical results of the time evolution of the cross-sectional area of the elastic tube of
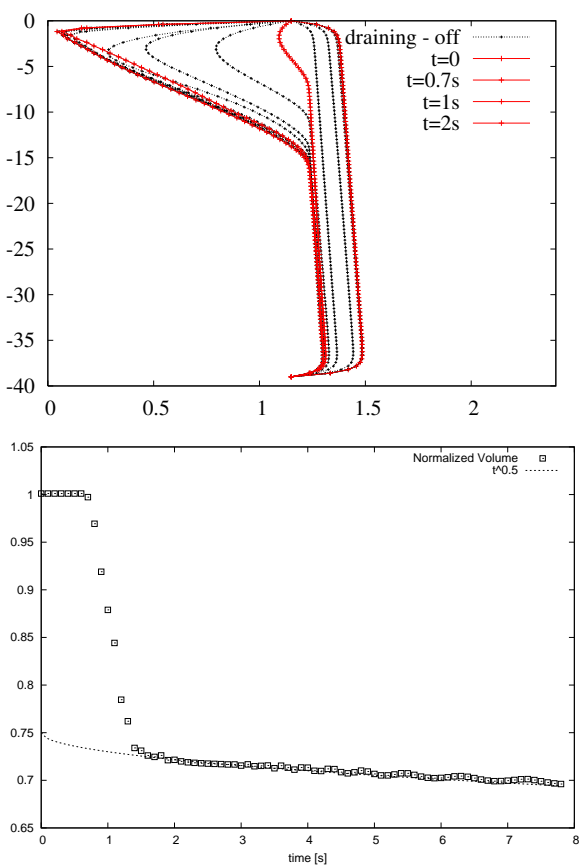

Fig. 9. (top) Area as function of time in a draining process. Close mode. (bottom) Normalized volume from the numerical data and scaling in $t^{1 / 2}$

a draining process in closed mode. We keep the definitions of Figure 8 to compare both tube dynamics, so we present the tube shape for constant time intervals of $0.1 \mathrm{~s}$ (black dotted lines) and the particular states at times 0.5, 0.7, 1 and 2 in red.

The first two phases of the collapse process are equivalent and after the second peak at time $t=1.1 \mathrm{~s}$ the upper zone of the tube is dominated by the viscous effects that determines the beginning of the viscous drainage, in which the fluid is locked at the lower part of the elastic tube and is slowly squeezed out. The length of the collapsed region increases very slowly and at time $t=10 \mathrm{~s}$ the elastic tube has lost less than $30 \%$ of its initial volume. Experimental evidence is given in Figure 5 (c) and numerical data are presented in Figure 9 (top). 


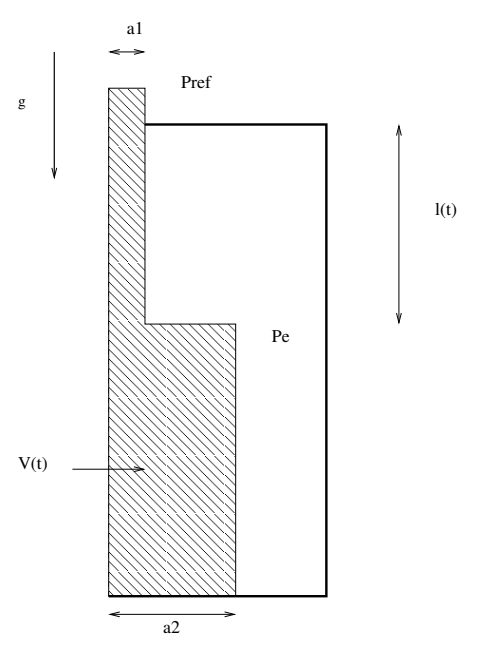

Fig. 10. Simple lumped model of the viscous process. A flexible tube of length $l(t)$ and cross-sectional area $a_{1}$ is connected to a volume $V(t)$ with characteristic cross-sectional area $a_{2}$. Under the external pressure $p_{e}(t)$ the length $l(t)$ moves down giving a rate of volume $-\frac{d V}{d t}$.

The solution of the numerical integration of the velocities of Figure 9 (top) presented in Figure 9 (bottom) gives the normalized tube volume, which is coherent with our lumped model prediction (c.f. end of this section) and shows that after a few seconds the residual volume is about $70 \%$, as experimentally observed.

We now attempt to model the asymptotic evolution of this volume variation. We roughly modeled the viscous process by using a lumped model consisting of a very viscous region of length $l(t)$ and constant area $a_{1}$ connected with an inflated region of constant area $a_{2}$ with initial volume $V_{i}$ (see Figure 10). The flow rate $Q$ of the system results from the balance between the pressure difference and the viscous forces under the assumption that the fluid flow can be treated as a Poiseuille flow in a circular tube. The momentum equation gives the simple governing equa- tion

$$
\frac{(\Delta p-\rho g l(t))}{l(t)} \sim K\left(a_{1}\right) \mu Q / a_{1}^{2}
$$

where $\mu$ is the coefficient of viscosity, $\Delta p=P_{e}-p_{a t m}$ is the external pressure drop acting on the collapsed part, $P_{e}$ is the external pressure and $p_{a t m}$ the outlet pressure at the top of the upper tube (the atmospheric pressure). The coefficient $K\left(a_{1}\right)$ depends on the shape of the tube cross-section, i.e., its value is $8 \pi$ for a circular tube. The temporal volume variation into the draining chamber is given by

$$
a_{2} \frac{d l}{d t}=-\frac{d V}{d t}
$$

The mass conservation gives $\frac{d V}{d t}=Q(t)$ and combining the last three equations we get the following differential equation for the time variation of the length $l(t)$,

$$
\frac{l(t)}{(\Delta p-g l(t))} \frac{d l}{d t}=-\frac{a_{1}^{2}}{K\left(a_{1}\right) \mu a_{2}} .
$$

Since $\Delta p>>g l(t)$, the scaling law for $l(t)$ follows

$$
l(t)^{2} \sim-\frac{2 . \Delta p a_{1}^{2}}{K\left(a_{1}\right) \mu a_{2}} t+c t e
$$

therefore $l$ scales as $t^{1 / 2}$, and finally, from equation (5), $Q \sim t^{-1 / 2}$ holds. This scaling law was already found in [11] from a non-linear diffusion equation combining moment and mass conservation.

Nevertheless, this simple model cannot explain the short time behavior for the following reasons: (i) for the small values of $l(t)$, the pressure drop cannot be approximated by the Poiseuille law, the length of the tube is too small to observe a fully developed flow; (ii) the quick variation of the flow rate from its peak to a lower value is not consistent with the assumption of a quasi-stationary flow, (iii) 
the section of the upper part of the tube cannot be considered as a constant.

Figure 9 (bottom) shows the time variation of the normalized volume obtained by integration of the numerical results presented in figure 9 (top). After the rapid transient, the system is governed by the viscous dynamics and the scaling law infers that the tube volume decreases as $t^{1 / 2}$ (doted line superposed over the normalized volume).

Note that there is no scaling plot for experimental data since the flow rate measurement technique does not allow to assess velocities lower than $2 \mathrm{~cm} / \mathrm{s}$.

\subsubsection{Filling open mode (pathological model)}

Figure 11 (a) shows the numerical shape of the crosssectional area from $1.2 s$ to the peak time in the flow rate (marked by the red line on the figure). We observe that the flow inflates progressively at both tube edges and that the cross-sectional area increases are well-correlated with the flow rate. We observe two dynamical fronts (from the bottom to the top and conversely) separating the inflated and the collapsed regions. When both fronts get in contact, the flow rate falls down and the elastic tube blows up rapidly reaching the final shape with some superimposed oscillations decaying in time. We can track the front dynamics as a function of time by plotting the successive positions of a specific point (in this case, for the reduced values cross-sectional area equal to 0.5). Figure 11 (b) presents the time evolution of these points starting from the top and the bottom of the elastic tube. The slope of the curves gives information about the front velocity: front velocities
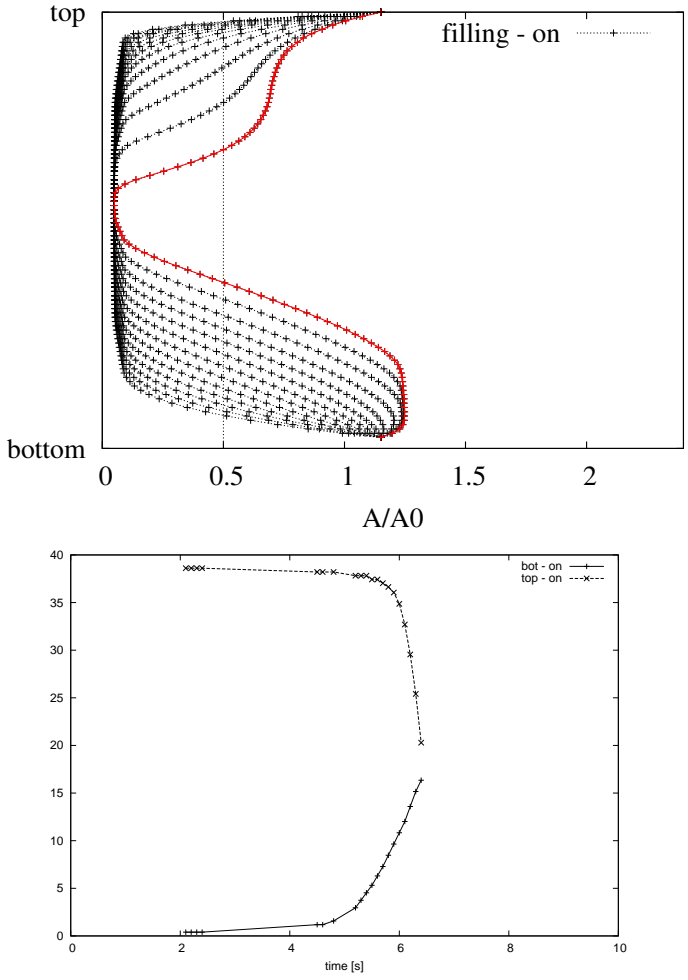

Fig. 11. (a) Numerical cross-sectional area as a function of time for a filling process in open mode. The red line marks the cross-sectional area at the flow rate peak. (b) Front dynamics: point trajectories into a space-time diagram of two particular cross-sectional area positions as a function of time

are not constant and both fronts accelerate right before contact.

The volume variation in the open filling mode (Figure $6(\mathrm{c}))$ is similar to that observed in the draining case (Figure $4(\mathrm{c}))$. We observe a saturation of the ejected volume that follows the flow rate peaks.

The numerical results of Figure 12 (a) show that the cross-sectional area shape of the filling process showed $n$ Figure 12 (a) moves exactly with the same time evolution as the upper area in Figure 11 (a). The peak in the flow rate appears when the front reaches the end side at the 

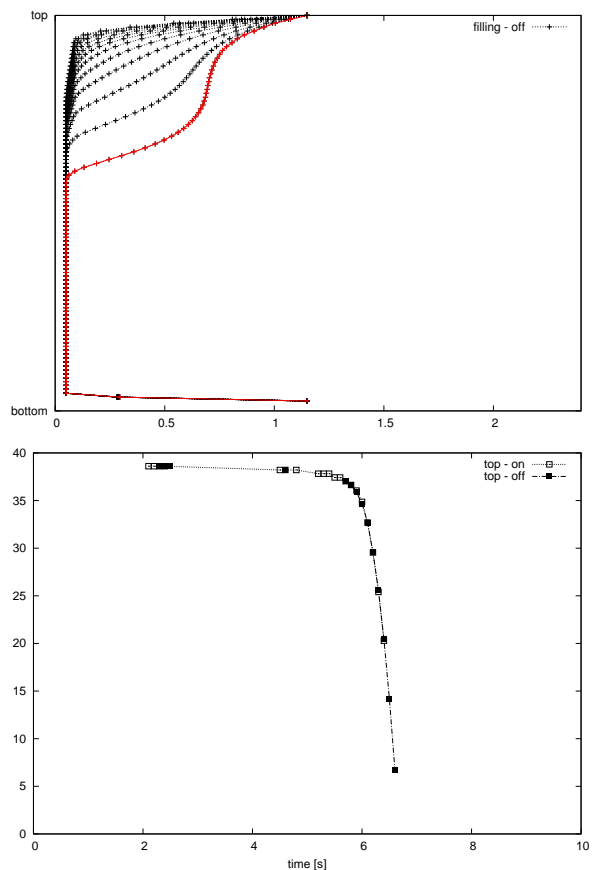

Fig. 12. (a) Numerical cross-sectional area as a function of time for a filling process in open mode. (b) Front dynamics: fixed cross-sectional area positions as a function of time for open and closed modes

bottom of the elastic tube. This travel difference explains the time shift between the peaks of the flow rate at the top exit (Figure $6(\mathrm{~b})$ ).

Figure 12 (b) superposes both front dynamics for the upper side (open and closed modes): observe that the two fronts coincide exactly, that is, until the information has not reached the other side (a front coming from the bottom or from the boundary conditions) the system behaves like in a semi-infinite configuration.

\subsubsection{Oscillations}

Experimental observations show an oscillating state at the end of the draining (open mode) as well as in the filling
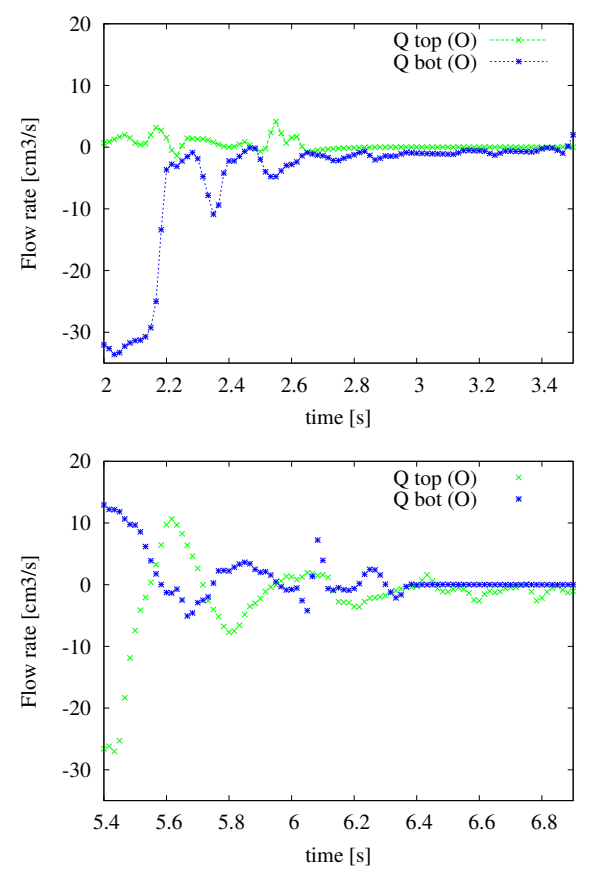

Fig. 13. Experimental data: detail of oscillating flow rates for (top) draining and (bottom) filling

(open and closed mode) process (the flow rate of Figures 4 and 6 are presented in a zoomed Figure 13).

The tube is either completely filled or empty and the fluid inside oscillates. The fluid is coupled with the hydraulic system composed by both chambers (top and bottom) and the secondary circuit. The hydraulic system consists of inertive-resistive elements and a capacitor. The flow peaks excite the coupled system and cause the replay at a frequency determined by the inertance and the capacitance of the elements.

A simple study of linear stability of the isolated collapsible tube allows to understand the phenomenon, we note that this study is relevant only if the wavelength is smaller than the typical length scale variation [26]. We start with the governing equations (1) and (2) where we replace $-f_{v}$ by $-R u$ for the sake of simplicity. By analysing 
the stability to the first order around an equilibrium position $\left(A_{0}, U_{0}, p_{0}\right)$ we find $A=A_{0}+\alpha, U=U_{0}+u$ and $p=p_{e}+p$, where $\alpha=A / A_{0}$. Replacing them in the governing equations we get a first approximation (dropping the quadratic terms):

$$
\begin{aligned}
& \alpha_{t}+U_{0} \alpha_{x}+A_{0} u_{x}=0 \\
& u_{t}+U_{0} u_{x}=-p_{x}-R u \\
& p=F^{\prime}\left(A / A_{0}\right) \alpha-T \alpha_{x x}
\end{aligned}
$$

Setting $F^{\prime}\left(A / A_{0}\right) \sim c_{0}, u_{1}=\hat{u} e^{i(k x-\omega t)}$ and $\alpha_{1}=$ $\hat{\alpha} e^{i(k x-\omega t)}$, and noting that the time derivatives are replaced by $-i \omega$ and the space derivatives by $i k$ we have the following system:

$$
\left(\begin{array}{cc}
k u_{0}-\omega & a_{0} k \\
k^{3} T+\frac{c_{0}^{2} k}{a_{0}} & -i R-\omega+k u_{0}
\end{array}\right)\left(\begin{array}{l}
\hat{\alpha} \\
\hat{u}
\end{array}\right)=\left(\begin{array}{l}
0 \\
\\
0
\end{array}\right)
$$

The necessary condition for a solution is that the determinant of the matrix is zero, thus giving the following complex equation for $\omega$ as a function of $k$ : for small $T$, they not longer exhibit the oscillatory behavior.

Even though one would logically expect a non oscillatory wave decay for $T \rightarrow 0$, numerical solutions show that it is observable only for the draining process; the filling process (open and close modes) decays always with finite oscillatory waves.

Such different dynamics could be explained by the ratio between the fluid volume of the elastic tube and of the hydraulic system (top and bottom chambers and secondary circuit). The final state for the filling process is an elastic inflated tube of a fluid volume of about $A * L \sim$ $58 \mathrm{~cm}^{3}$. That volume is large enough and then, the added mass excites the hydraulic system and dominates the oscillatory behavior. In addition, there are very small viscous effects on the elastic tube.

Conversely, in the draining process, the final volume is around 30 times smaller because the tube is collapsed (hence with high viscous effects). Under such condition, the empty tube could be considered independent from the hydraulic system and the stability analysis holds.

\section{Conclusion}

The imaginary part of $\omega, \omega_{i}$, governs the wave dynamics; waves will grow or decay with $\omega_{i}>0$ or $\omega_{i}<0$. For $\mathrm{T} \neq 0$ and $\mathrm{R} \neq 0$ we have

$$
\frac{\omega}{k}=u_{0} \pm \sqrt{c_{0}^{2}+a_{0} k^{2} T-\frac{R^{2}}{2 k^{2}}}-i \frac{R}{2 k}
$$

Therefore, the longitudinal tension $T$ is in competition with the frictional forces $R$. The waves decay in time and,
We have developed a complex experimental setup modeling the venous flow through muscular veins. The optical reconstruction technique allows to evaluate with good precision the cross-sectional area of the elastic tubes. Comparisons made with a classical ombroscopy reconstruction show that our approach improves the results, especially in the narrow sections when the elastic tube is collapsed. 
The evaluation of the cross-sectional area in that situation is crucial to understand the dynamical behavior of the viscous dynamics. On the other hand, since the numerical simulation depends on the quality of the experimental tube law, for high negative transmural pressure, numerical simulations using the ombroscopy results cannot correctly describe the viscous dynamics.

The numerical results from a simple 1D model led to a good understanding of the experimental data, which are quite complex. We recall that the numerical model is parameter free and is able to follow the flow dynamics at any time (peaks, total volume, scaling).

Comparisons between experimental and numerical data show that the ejected volume is roughly the same in open and closed modes when the tube is draining (see Figures $4(\mathrm{c})$ and $5(\mathrm{c}))$. This means that the valve behavior has little influence, due perhaps to the tube length $(\sim 40 \mathrm{~cm})$; instead, for physiological lengths (i.e. shorter than $40 \mathrm{~cm}$ ), it is possible that the wave reflections and back flows hide that phenomenon. Moreover, in drainingfilling cycles as in breath or muscular pumping, the valve failure is only actually seen at the end of the process (recall the AVP definition in the introduction), so it seems that these facts have to be correlated with the physiological data.

We have also presented a lumped model that describes the viscous dynamics of the system, in particular the scaling law of the volume dynamics $(\sim 1 / 2)$. In the front dynamics for the upper side (open and closed modes) we remark that the two fronts superpose. Consequently, there is a short time where the system behaves like an infinite tube as expected in a wave-like system. Finally, a stability analysis allows to find the experimental observations: when the volume of the secondary circuit is large enough the added mass excites the hydraulic system that dominates the oscillatory behavior; the viscous effects on the elastic tube are not important enough to slow down the perturbation.

\section{References}

1. A. H. Shapiro. Steady flow in collapsible tubes. Asme J. Biomech. Eng., 1977.

2. R. D. Kamm. Bioengineering studies of periodic external compression as prophylaxis against deep vein thrombosis - Part I: Numerical studies. J. Biomech. Eng., 1982.

3. D. L. Jan, R. D. Kamm, and A. H. Shapiro. Filling of partially collapsed compliant tube. J. Biomech. Eng., 1983.

4. D. Elad, R. D. Kamm, and A. H. Shapiro. Choking phenomena in a lung like model. ASME J. Biomech. Eng., 1987.

5. D. J. Griffiths. Hydrodynamics of male micturation. I. theory of steady flow through elastic walled tubes. Med. Biol. Eng., 1971.

6. D. F. Young and F. Y. Tsai. Flow characterists in models of arterial stenosis. I. Steady flow. J. Biomech., 1975.

7. M. E. McClurken, I. Kececioglu, R. D. Kamm, and A. H. Shapiro. Steady, supercritical flow in collapsible tubes. Part 2. Theoretical studies. J Fluid Mech, 109:391-415, 1981.

8. R. D. Kamm and T. J. Pedley. Flow in collapsible tube: a brief review. J. Biomech. Eng., 1989. 
9. W. A. Conrad. Pressure-flow relationships in collapsible tubes. IEEE Transactions on Biomedical Engineering, 1969.

10. J. P. Holt. Flow through collapsible tubes and through in situ veins. IEEE Trans. Bio-Med. Engng., 1969.

11. R. D. Kamm and A. H. Shapiro. Unsteady flow in a collapsible tube subjected to external pressure of body forces. J. Fluid Mech., 1979.

12. D. A. Olson, R. D. Kamm, and A. H. Shapiro. Bioengineering studies of periodic external compression as prophylaxis against deep vein thrombosis - Part II: Experimental studies on a simulated leg. J. Biomech. Eng., 1982.

13. C. Cancelli and T. J. Pedley. A separated flow model for collapsible tube oscillations. J. Fluid Mech., 1985.

14. Y. Matsuzaki and T. Matsumoto. Flow in a twodimensional collapsible channel with rigid inlet and outlet. ASME J. Biomech. Eng., 1989.

15. T. Ikeda and Y. Matsuzaki. A one-dimensional unsteady separable and reattachable flow model for collapsible tubeflow analysis. J. Biomech. Eng., 1999.

16. C. D. Bertram and M. D. Sheppeard. Interactions of pulsatile upstream forcing with flow-induced oscillations of a collapsed tube: mode-locking. Med Eng Phys, 22(1):29-37, Jan 2000 .

17. C. D. Bertram, J. Timmer, T. G. Mller, T. Maiwald, M. Winterhalder, and H. U. Voss. Aperiodic flow-induced oscillations of collapsible tubes: a critical reappraisal. Med Eng Phys, 26(3):201-14, Apr 2004.

18. C. D. Bertram. Fluid flow in distensible vessels. Clin. and Exp. Pharm. and Phys., 36(2):206-216, 2009.

19. C. D. Bertram, G. Diaz de Tuesta, and A. H. Nugent. Laser-Doppler measurements of velocities just downstream of a collapsible tube during flow-induced oscillations. $J$ Biomech Eng, 123(5):493-9, Oct 2001.

20. Y. C. Fung. Biomechanics: Mechanical Properties of Living Tissues. Springer-Verlag. New York, 1993.

21. P. Guesdon. PhD thesis, University Paris 7, 2006.

22. C. Ribreau, S. Naili, and A. Langlet. Head losses in smooth pipes obtained from collapsed tubes. J. Fluids Struc., 8:183-200, 1994.

23. XY Luo and TJ Pedley. The effects of wall inertia on flow in a two-dimensional collapsible channel. Journal of Fluid Mechanics, 363:253-280, 1998.

24. D. Elad, D. Katz, E. Kimmel, and S. Einav. Numerical schemes for unsteady fluid flow through collapsible tubes. J Biomed Eng., 13:10-18, 1991.

25. J.-M. Fullana, F. Cros, P. Flaud, and S. Zaleski. Filling a collapsible tube. Journal of Fluid Mechanics, 494:285-296, 2003.

26. O. E. Jensen. Instabilities of flow in a collapsed tube. $J$ Fluid Mech., 1990. 